\title{
Nanoparticles as biochemical sensors
}

This article was published in the following Dove Press journal:

Nanotechnology, Science and Applications

22 September 2010

Number of times this article has been viewed

\section{Afaf El-Ansary Layla M Faddah}

Biochemistry Department, Science College, and Pharmacology

Department, Pharmacy College, King Saud University, Riyadh, Saudi Arabia

Correspondence: Afaf El-Ansary PO Box 22452, King Saud University, Riyadh I I495, Saudi Arabia

Tel + 966 I 4682184

Fax + 966 | 4769137

Email elansary@ksu.edu.sa
Abstract: There is little doubt that nanoparticles offer real and new opportunities in many fields, such as biomedicine and materials science. Such particles are small enough to enter almost all areas of the body, including cells and organelles, potentially leading to new approaches in nanomedicine. Sensors for small molecules of biochemical interest are of critical importance. This review is an attempt to trace the use of nanomaterials in biochemical sensor design. The possibility of using nanoparticles functionalized with antibodies as markers for proteins will be elucidated. Moreover, capabilities and applications for nanoparticles based on gold, silver, magnetic, and semiconductor materials (quantum dots), used in optical (absorbance, luminescence, surface enhanced Raman spectroscopy, surface plasmon resonance), electrochemical, and mass-sensitive sensors will be highlighted. The unique ability of nanosensors to improve the analysis of biochemical fluids is discussed either through considering the use of nanoparticles for in vitro molecular diagnosis, or in the biological/biochemical analysis for in vivo interaction with the human body.

Keywords: nanoparticles, quantum dots, nanomedicine, biochemical sensors, antibodies, in vivo, in vivo

\section{Introduction}

There are well-developed methods to synthesize nanomaterials (eg, nanorod, ${ }^{1}$ nanocube, ${ }^{2}$ nanoshell, ${ }^{3}$ which have found real applications in practice. The light-scattering properties of new nanomaterials ${ }^{4}$ in different composition, size, and shape have greatly attracted the attention of analysts, so the applications of nanomaterials for analytical purposes have grown dramatically. Because of their unique advantages, nanoparticles (NPs), with colorful light-scattering properties, are comparable to optical probes with various fluorescent dyes. For example, the light-scattering power of a single NP label is in order of magnitude greater than a single fluorescent label, and the light signals generated from these nanomaterials are not prone to photobleaching, even under illumination for a long time. What is more, in light-scattering detection, only a simple instrument is required, compared with a fluorescent system. ${ }^{5}$

Metal NPs with large diameter $(>30 \mathrm{~nm})$ exhibit strong light-scattering in the visible region, which could be used directly for light-scattering labels in biochemical assay. However, small NPs, which apparently do not feature light-scattering, can also be used to sense chemical interactions (eg, antigen-antibody, avidin-biotin, DNA hybridization, and electrostatic attraction), since enhanced light-scattering signals would be produced if these NPs were to aggregate during the interactions. Because 
the enhanced light-scattering signals from the aggregated species are sufficiently sensitive to monitor NP aggregation, in a simple procedure, biochemical assay based on such light-scattering signals has been widely used in the determination of DNA, proteins, and drugs. ${ }^{5}$

Immunoassay is important in basic research and clinical diagnostics. Sandwich-type immunoassay, using a primary antibody to capture the analyte, and a labeled, secondary antibody to detect antigen binding, is widely accepted procedure. The aggregation of nanomaterials, especially gold ( $\mathrm{Au}$ ), induced by the immunoreactions offers a new approach for immunoassay, ${ }^{6}$ using light-scattering detection to obtain high sensitivity. The enhanced light-scattering signals from the AuNP aggregates, induced by the immunoreaction between the apolipoprotein and its AuNP-labeled antibody, have been successfully applied in clinical diagnostics. ${ }^{7}$ There have been similar reports about light-scattering signals involving such different materials as silver nanoparticles (AgNP) and analytes (eg, fibrinogen and human immunoglobulin $\mathrm{G}[\mathrm{h}-\mathrm{IgG}]){ }^{8}$ Interactions between proteins and nanomaterials have been applied in quantitative assays of proteins in biochemistry and clinical diagnostics. Electrostatic attraction between proteins and nanomaterials with opposite charge induces assembly of proteins or nanomaterials, resulting in enhanced light-scattering signals. Various nanomaterials (either metal or nonmetal), functionalized with the opposite charge to the protein, have been used to detect protein quantitatively with ng sensitivity.

This information stimulates our interest to review major research work related to the use of nanoparticles as biochemical sensors, and to highlight the advantages of using this strategy over the common analytical methods.

\section{Nanoparticles in analytical biochemistry}

One of the most important functions of nanoparticles is catalysis, especially with noble metal nanoparticles, which have high catalytic activity for many chemical reactions. Because nanomaterials also have good biocompatibility, they are used to immobilize biomolecules for the fabrication of biosensors.

\section{Glucose nanosensors}

Glucose is a key metabolite for living organisms, especially in the case of patients suffering diabetes. Since the first enzyme electrode was reported in 1962 by Clark and Lyons. ${ }^{9}$ There has been an increasing demand for the development of new methodologies for a rapid, simple, reliable, reproducible, and sensitive determination of glucose. The detection of glucose by electrochemical biosensors is based on the electrochemical oxidation of hydrogen peroxide $\left(\mathrm{H}_{2} \mathrm{O}_{2}\right)$ generated by enzyme-catalyzed oxidation of glucose at anodic potentials $(\mathrm{N}+0.6 \mathrm{~V}$ vs $\mathrm{Ag} / \mathrm{AgCl}) .{ }^{10}$ The immobilization of glucose oxidase (GOx) on the electrode surface, which is one of the main factors that affects the performance of a glucose biosensor, has received considerable attention in recent years. ${ }^{11}$ A new amperometric glucose biosensor was constructed, based on the immobilization of GOx with crosslinking in a matrix of chitosan (CHIT) on a glassy carbon electrode (GCE), which was modified by layer-by-layerassembled carbon nanotube (CNT)/CHIT/gold nanoparticle (GNP) multilayer films. With the increasing of CNT/CHIT/ GNP layers, the response current to $\mathrm{H}_{2} \mathrm{O}_{2}$ changed regularly, and the response current reached a maximum value when the number of CNT/CHIT/GNP layers was 8 . The assembly process for the multilayer films was simple to operate. With GOx as an enzyme model, a new glucose biosensor was fabricated. The excellent electocatalytic activity and special structure of the enzyme electrode resulted in a detection limit of $3 \times 10^{-6} \mathrm{M}$, estimated at a signal-to-noise ratio of 3 , at a fast response time (less than $6 \mathrm{~s}$ ). Moreover, it exhibited good reproducibility and stability. Human plasma samples were assayed in order to demonstrate the practical usage of the biosensor. Fresh plasma sample was first analyzed in the local hospital with the ASCA AGII Chemistry System (Landmark Scientific, Greensboro, NC). The samples were then re-assayed with the CNT/CHIT/GNP)8/GOx/GCE. A plasma sample $(0.5 \mathrm{~mL})$ was added into $2 \mathrm{~mL}$ of 0.067

Table I Performances of some NADH sensors

\begin{tabular}{llll}
\hline NADH sensor method & Linear range & Detection limit & Reference \\
\hline GC/AuNP/amperometry & $1.25 \times 10^{-6} \mathrm{M}$ to $3.08 \times 10^{-4} \mathrm{M}$ & $2.5 \times 10^{-7} \mathrm{M}$ & 23 \\
ITO/DA-TiO,/Pliotoelectrochemical & $5.0 \times 10^{-7} \mathrm{M}$ to $1.2 \times 10^{-4} \mathrm{M}$ & $1.4 \times 10^{-7} \mathrm{M}$ & 19 \\
CNF-CPE/amperometry & $1.15 \times 10^{-7} \mathrm{M}$ & $0.2 \times 10^{-7} \mathrm{M}$ & 24 \\
Pd-AuNP/spectroscopic & $1.6 \times 10^{-4} \mathrm{M}$ & $4.5 \times 10^{-5} \mathrm{M}$ & 24 \\
\hline
\end{tabular}

Abbreviations: $\mathrm{NADH}$, dihydronicotinamide adenine dinucleotide; GC, glassy carbon electrode; AuNP, gold nanoparticles; ITO, indium-tin-oxide; DA, dopamine; $\mathrm{TiO}_{2}$, titanium dioxide; $\mathrm{CNF}$, carbon nanofibers; $\mathrm{CPE}$, carboxypeptidase $\mathrm{E}$; Pd, palladium; $\mathrm{M}$, mole. 
M phosphate buffer saline ( $\mathrm{pH} 7.0$ ) and the response was obtained at $+0.6 \mathrm{~V}$. The contents of glucose in blood could then be calculated from the calibration curve. The results are satisfactory and agree closely with those measured by the biochemical analyzer in the hospital. ${ }^{11}$

Recently, platinum nanoparticles (PtNPs) were deposited on mesoporous carbon material (CMK-3). GOx was immobilized in the resulting Pt nanoparticles/mesoporous carbon (Pt/CMK-3) matrix, and then the mixture was cast on a glassy carbon electrode using gelatin as a binder. The glucose biosensor exhibited excellent current response to glucose after cross-linking with glutaraldehyde. At $0.6 \mathrm{~V}$ (vs saturated calomel electrode) the response current was linear to glucose concentration in the range of $0.04-12.2 \mathrm{mM}$. The response time (time for achieving 95\% of the maximum current) was $15 \mathrm{~s}$ and the detection limit (signal-to-noise ratio =3) was $1 \mu \mathrm{M}$. The Michaelis-Menten constant $\left(\mathrm{Km}^{\text {app }}\right)$ and the maximum current density were $10.8 \mathrm{mM}$ and $908 \mu \mathrm{Acm}^{-2}$ respectively. The activation energy of the enzymatic reaction was estimated to be $22.54 \mathrm{kJmol}^{-1}{ }^{12}$ The biosensor showed good stability. It achieved the maximum response current at about $52^{\circ} \mathrm{C}$ and retained $95.1 \%$ of its initial response current after being stored for 30 days. In addition, some fabrication and operational parameters for the biosensor were optimized in this work. The biosensor was used to monitor the glucose levels of serum samples after being covered with an extra Nafion film to improve its anti-interferent ability, and satisfactory results were obtained. The interference of some electroactive compounds to the glucose response was examined by Yu et al. ${ }^{12}$ When the glucose concentration was $5.6 \mathrm{mM}$, the deviation of response was calculated according to (Ii- $\mathrm{Ig}) / \mathrm{Ig}$, where Ii and Ig were the steady-state currents recorded for solutions containing glucose and interferent, or glucose alone. At Pt/CMK-3-GOx-gelatin/GCE, the deviations caused by ascorbic acid (AA) (0.1 mM), p-acetaminophenol (0.05 mM) and uric acid (UA) $(0.5 \mathrm{mM})$ were $0.44 \%, 4.69 \%$, and $13.2 \%$ respectively. This indicates that the biosensor suffersed some influence from coexistents in serum samples. Therefore, $1 \mu \mathrm{M}$ percentage by weight Nafion was cast on the surface of $\mathrm{Pt} /$ CMK-3-GOx-gelatin/GCE to prevent the interference. And then, at Nafion/Pt/CMK-3-GOx-gelatin/GCE, the deviations were in the acceptable range $( \pm 10 \%) .{ }^{12}$

A silver hexacyanoferrate nanoparticle (AgHCFNP)/CNT modified GCE was fabricated and then successfully used for the simultaneous determination of AA, UA, and dopamine (DA) by cyclic voltammetry $(\mathrm{CV})$. Meissam et $\mathrm{al}^{13}$ reported for the first time a simultaneous determination of AA, DA, and UA using a glassy carbon electrode modified with a mixture of AgHCFNPs and multiwalled carbon nanotubes. Compared with the existing reports about simultaneous determination of AA, DA, and UA, the proposed method is much more convenient, as to preparation of the GCE/CNTAgHCFNP-modified electrode.

The voltametric behaviors of a mixture of AA, DA, and UA on bare GCE, GCE/CNT, and GCE/CNT-AgHCFNP elctrodes were studied, and a comparison of the $\mathrm{CV}$ results is shown in Figure 1. DA showed broad oxidation peak and could not be discriminated from AA and UA when a bare GC electrode was used (Figure 1A). For DA, the anodic and cathodic peaks appear at $0.482 \mathrm{~V}$ and $0.552 \mathrm{~V}$, respectively, and the separation of peak potential is about $0.070 \mathrm{~V}$. Moreover, the cathodic peak current is much smaller than the anodic peak current. It is clear that the electrochemical reaction of DA at the bare GCE is irreversible, indicating the sluggish electron transfer kinetic of DA at the bare GCE, which may be related to electrode fouling caused by the deposition of this compound and its oxidation product on the electrode surface. ${ }^{14}$ When GCE/CNT or GCE/CNT-AgHCFNPs was used as the working electrode, the detection sensitivity was improved significantly, and effective separation of the anodic peaks of AA, DA, and UA was obtained. ${ }^{13}$

It could be suggested that the modification of the GCE surface by CNT-AgHCFNPs not only improves the electrochemical catalytic activities towards the oxidation of AA, DA, and UA, but also resolves the overlapped oxidation peaks of AA, DA, and UA into three well-defined peaks at potentials $0.193 \mathrm{~V}, 0.444 \mathrm{~V}$, and $0.620 \mathrm{~V}$ in $\mathrm{CV}$, respectively. Comparison between GCE/CNT and GCE/CNT-AgHCFNPs demonstrates that GCE/CNT-AgHCFNPs better facilitates the simultaneous determination of AA, DA, and UA with good stability, sensitivity, and selectivity. In comparison with other electroanalytical methods that report for the simultaneous determination of ascorbic acid, dopamine, and uric acid, this method has better figures of merit. The proposed method can be applied to the simultaneous determination of AA, DA, and UA concentrations in real samples with satisfactory results. ${ }^{13}$

\section{Choline nanosensors}

Choline is distributed in the central and peripheral nervous systems of mammals. ${ }^{15}$ It is also an important component of phospholipids (lecithin and sphingomyelin), which are required for the synthesis of the neurotransmitter precursor acetylcholine. ${ }^{16}$ Classified as vitamin-like, choline is generally uptaken by the human body from both dietary and endogenous sources. Determination of choline is carried out 


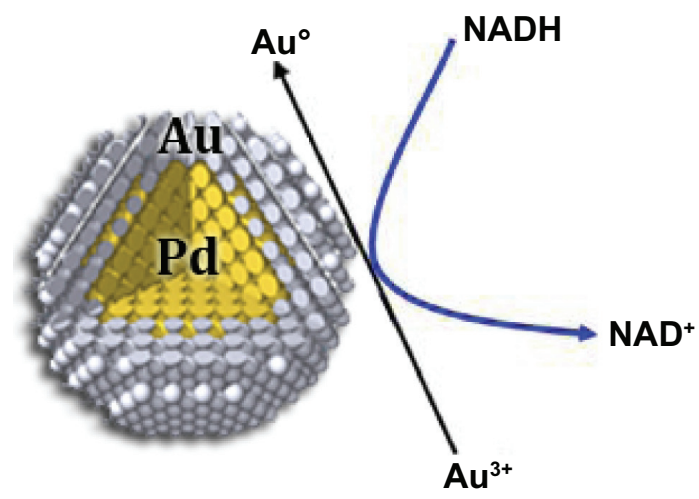

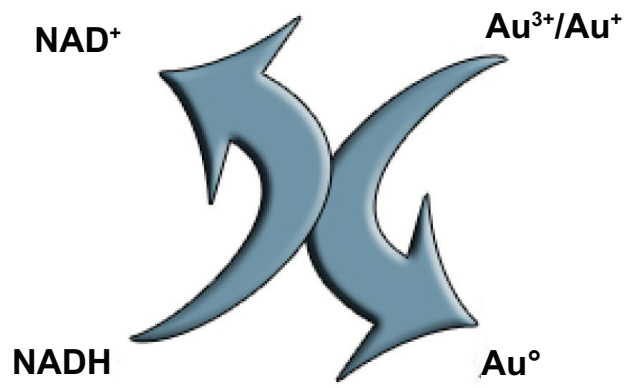

$\mathrm{Au}^{+}(\mathrm{OH})$ or $\mathrm{Au}^{3+}(\mathrm{OH})_{3}$

Figure I Proposed mechanism for Pd (core)-Au (shell) NPs catalyzed oxidation of NADH to NAD. ${ }^{24}$

Abbreviations: NADH, dihydronicotinamide adenine dinucleotide; NAD+, nicotinamide adenine dinucleotide; NPs, nanoparticles; Au, gold; Pd, palladium.

by a number of techniques nowadays. As the most promising alternative, enzyme-based biosensors have emerged in the past few years for direct monitoring of choline. A number of enzyme electrodes, with immobilized ChOx, were reported for choline determination based on the detection of liberated $\mathrm{H}_{2} \mathrm{O}_{2} \cdot{ }^{17,18}$ The manganese dioxide $\left(\mathrm{MnO}_{2}\right)$ nanoparticlemodified electrodes show a bidirectional electrocatalytic ability toward the reduction/oxidation of $\mathrm{H}_{2} \mathrm{O}_{2}$. Based on this property, a choline biosensor was fabricated via direct and facile electrochemical deposition of a biocomposite that was made of chitosan hydrogel, $\mathrm{ChOx}$, and $\mathrm{MnO}_{2}$ nanoparticles onto a glassy carbon electrode. The biocomposite is homogeneous and easily prepared, and provides a shelter for the enzyme to retain its bioactivity. The results of square wave voltammetry showed that the electrocatalytic reduction currents increased linearly with the increase of choline chloride concentration in the range $1.0 \times 10^{-5} \mathrm{M}-2.1 \times 10^{-3} \mathrm{M}$, and no obvious interference from AA and UA was observed. Good reproducibility and stability were obtained.

Carbon nanotube-modified electrodes have shown excellent electrocatalytic activity towards some electromotive substances, such as $\mathrm{H}_{2} \mathrm{O}_{2}$, due to the fast electron transfer ability of CNTs. ${ }^{19}$

\section{Nicotinamide adenine dinucleotide nanosensors}

Nicotinamide adenine dinucleotide $\left(\mathrm{NAD}^{+}\right)$and dihydronicotinamide adenine dinucleotide (NADH) are the key central charge carriers in living cells. $\mathrm{NAD}^{+}$is a ubiquitous cofactor utilized by more than 300 dehydrogenase enzymes. The nicotinamide region is the site of reversible redox processes in living cells. $\mathrm{NAD}^{+}$and phosphorylated $\mathrm{NAD}^{+}\left(\mathrm{NADP}^{+}\right)$ are the two forms of coenzyme. NADH and NADPH are reduced forms of $\mathrm{NAD}^{+}, \mathrm{NADP}^{+}$respectively. While $\mathrm{NAD}^{+}$ and $\mathrm{NADP}^{+}$accept electrons from other molecules, NADH and NADPH donate electrons. NADH plays a key role in the multi-enzyme redox system in the microchrondrial transport chain. ${ }^{20} \mathrm{~A}$ large amount of byproduct is often produced during the direct electrode process. Also, the adsorbed $\mathrm{NAD}^{+}$could cause electrode fouling. Further, the high potentials used for the oxidation of NADH could also cause simultaneous oxidation of many interfering substrates, such as AA. ${ }^{21}$

The sensitivity of detection of NADH was determined from the plot of absorbance (at $340 \mathrm{~nm}$ ) vs $\mathrm{C}_{\mathrm{NADH}}$. Typical calibration plots were deduced from ultraviolet visible spectroscopy (UV-vis) recorded at $10 \mathrm{~min}$ and $90 \mathrm{~min}$. The sensitivity of NADH was determined to be $0.45 \mathrm{mM}$ per unit of absorbance. The detection limit of NADH was estimated to be $0.45 \mu \mathrm{M}$. The spectrophotometer can measure absorbance with an accuracy of 0.001 . Because the sensitivity of detection for NADH is $0.45 \mathrm{mM}$ for unit absorbance change, the detection limit for NADH could be $0.45 \mu \mathrm{M}$. Nanoparticle-NADH sensors performances are different depending on the composition of the sensor, showing different detection limits and linearity. ${ }^{22,23}$

A plausible mechanism for Palladium (Pd) (core)-Au (shell) NP-catalyzed conversion of NADH is proposed by Gopalan et al (2009). ${ }^{24}$ It is clear that the position of surface plasmon resonance (SPR) bands of AuNPs showed significant shift during the catalytic transformation of NADH to $\mathrm{NAD}^{+}$. It is therefore envisioned that the surface of the Au layer catalyzes the reaction. They presume that oxygen molecules adsorbed on the surface of the Au layer may induce oxidation of NADH to $\mathrm{NAD}^{+}$. It is known that reactions such as low 
temperature oxidation of carbon monoxide or propylene that involve oxygen as oxidant are catalyzed by AuNPs. ${ }^{25}$ In the Gopalan model, ${ }^{24} \mathrm{Au}^{+}$or $\mathrm{Au}^{3+}$ species are expected to present in the shell layer ( $\mathrm{Au}$ ) of the $\mathrm{Pd}$ (core)-Au (shell) catalyst. NADH oxidation was facilitated by the conversion of $\mathrm{Au}^{3+} /$ $\mathrm{Au}^{+}$to $\mathrm{Au}^{0}$ (Figure 1).

\section{Lactate nanosensors}

L-lactate is constantly produced from pyruvate by lactate dehydrogenase (LDH) in a process of fermentation during normal metabolism and exercise. L-lactate concentration plays an important role in clinical diagnostics, medicine validation, and food analysis. For example, the concentration of L-lactate in blood is a fundamental parameter for the prevention and diagnosis of a number of clinical disorders such as hypoxia ${ }^{26,27}$ some acute heart diseases, and drug toxicity tests. Over the past few years, various approaches based on LDH or lactate oxidase have been developed for the detection of lactate. They employ different methods to attach enzymes on the sensing layer, including adsorption, ${ }^{28}$ cross-linking, ${ }^{29}$ covalent attachment, ${ }^{30,31}$ conducting polymer entrapment, ${ }^{32,33}$ and confinement in sol-gel matrix. ${ }^{34}$ Flow injection analysis ${ }^{35,36}$ and mediator- based lactate biosensors ${ }^{37}$ have also been developed. In the LDH-based amperometric biosensor, LDH catalyzes the oxidation of lactate to pyruvate in the presence of the oxidized form of $\mathrm{NAD}^{+}$.However, the LDH-based biosensors have some common drawbacks, including the fact that they are unstable and that the electrochemical oxidation of the reduced form of NAD commonly occurs at high potentials, which can allow interference from other electroactive compounds usually present in real samples. Moreover, the intermediates produced during the oxidation reaction can lead to electrode fouling. Therefore, there is a demand for the development of a biosensor that is stable, reproducible, and sensitive for lactate detection in real samples. Recently, Rahman et $\mathrm{al}^{138}$ developed a lactate biosensor based on a conducting polymer, poly-5, 20-50,200-terthiophene-30-carboxylic acid (pTTCA), and multiwall carbon nanotube (MWNT) composite on a gold electrode. $\mathrm{LDH}$ and the oxidized $\mathrm{NAD}^{+}$were subsequently immobilized onto the pTTCA/MWNT composite film. Analytical performances and dynamic ranges of the sensor were determined, and the results showed that the sensitivity, stability, and reproducibility of the sensor improved significantly using the pTTCA/MWNT composite film. The calibration plot was linear (coefficient of determination $=0.9995$ ) over the range of 5 to $90 \mu \mathrm{M}$. The sensitivity was approximately $0.0106 \mu \mathrm{A} /$ $\mu \mathrm{M}$, with a detection limit of $1 \mu \mathrm{M}$, based on a signal-to-noise ratio of 3. The applicability of the sensor for the analysis of L-lactate concentration in commercial milk and human serum samples was demonstrated successfully.

\section{Triglyceride nanosensors}

Estimation of triglycerides (triacylglycerols) is extremely important, since its high concentration (normal range: $40-160 \mathrm{mg} / \mathrm{dL}$ in men, and $35-135 \mathrm{mg} / \mathrm{dL}$ in women) can cause hyperlipidemia. ${ }^{39-41}$ Apart from coronary diseases, hyperlipidemia is associated with several disorders, including diabetes mellitus, nephrosis, liver obstruction, and endocrine impairment. Moreover, estimation of triglyceride content in food has become important due to increased health awareness and stringent regulatory laws. ${ }^{42}$ The conventional methods for triglycerides detection, such as colorimetric or fluorometric techniques, are based on enzymatic hydrolysis of triglycerides to glycerol and free fatty acids. ${ }^{43-45}$ Most triglyceride biosensors reported to date are based on multi-enzymes, wherein a biochemical reaction depends upon the enzyme kinetics of another enzymatic reaction. McGowan et $\mathrm{al}^{46}$ have described an enzymatic method for serum triglyceride detection that involves simultaneous catalysis of four bio-enzymes (lipase, glycerol kinase, L-a-glycerophosphate oxidase, and peroxidase). ${ }^{46}$ These methods are, however, time-consuming, complicated, and expensive. Recently, lipase has been immobilized onto a sol-gel-derived nanostuctured cerium oxide (Nano- $\mathrm{CeO}_{2}, 35 \mathrm{~nm}$ ) film deposited onto indium-tin-oxide(ITO) coated glass plate for tributyrin detection. ${ }^{47}$ The Nano$\mathrm{CeO}_{2} / \mathrm{ITO}$ electrode and lipase/nano- $\mathrm{CeO}_{2} / \mathrm{ITO}$ bioelectrode have been characterized using scanning electron microscopy (SEM) and CV. The electrochemical response of the lipase/ nano- $\mathrm{CeO}_{2} /$ ITO bioelectrode towards tributyrin, investigated using CV studies, exhibits linearity, detection limit, and shelf life as $50-500 \mathrm{mg} / \mathrm{dl}, 32.8 \mathrm{mg} / \mathrm{dl}$, and 12 weeks respectively. The value of the apparent Michaelis-Menten constant obtained as $22.27 \mathrm{mg} / \mathrm{dl}(0.736 \mathrm{mM})$ for the lipase/ nano- $\mathrm{CeO}_{2} /$ ITO bioelectrode, indicating a high affinity of lipase with tributyrin samples (Figure 2). ${ }^{47}$

\section{Ochratoxin A detection}

Among the various metal oxides, nanostructured zinc oxide $(\mathrm{ZnO})$ has been used for immunosensor applications. ${ }^{48-51}$ Its unique properties, such as high isoelectric point $($ IEP $\approx 9.5$ ) and biocompatibility, facilitate immobilization of an enzyme and protein having low IEP via electrostatic interactions. Besides this, the positively charged $\mathrm{ZnO}$ nanoparticles not only provide a friendly microenvironment for immobilizing negatively charged rabbit antibodies ( $\mathrm{r}$-IgGs; IEP $\approx 5.5$ ) 


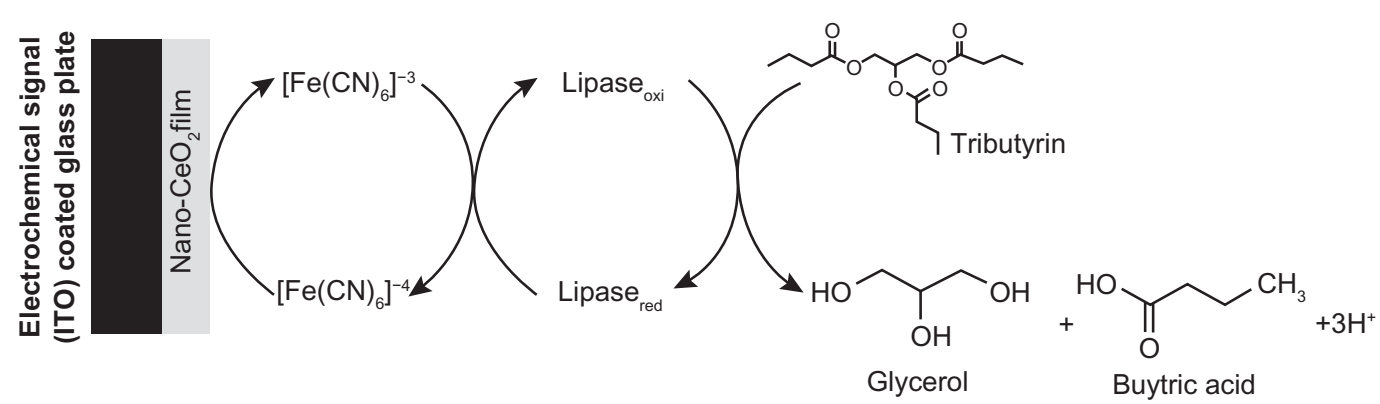

Figure 2 Electrochemical reaction at lipase/nano- $\mathrm{CeO}_{2} / \mathrm{ITO}$ bioelectrode. ${ }^{47}$ Abbreviations: $\mathrm{CeO}_{2}$, cerium oxide; ITO, indium-tin-oxide.

and retain bioactivity, but also accelerate electron transfer communication between the protein and the electrode to a large extent. ${ }^{48,49}$ Moreover, nontoxicity, high chemical stability, and high electron transfer capability make Nano-ZnO a promising material for immobilization of desired biomolecules for fabrication of an immunosensor. The sol-gel derived Nano-ZnO has recently emerged as an attractive material due to its ease of preparation under ambient conditions, tunable porosity, high thermal stability, chemical inertness, and negligible swelling in aqueous and nonaqueous solutions for immobilization of desired biomolecules (enzymes, proteins, and antibodies) ${ }^{52}$ Liu et $\mathrm{al}^{51}$ have prepared stable, nanosized, flower-like $\mathrm{ZnO}$ film by a hydrothermal method for a $\mathrm{H}_{2} \mathrm{O}_{2}$ sensor. $\mathrm{ZnO}$ nanocomb has been fabricated in bulk quantity, by the vapor phase transport method, for glucose detection. ${ }^{48}$ Wei et $\mathrm{al}^{48}$ have grown $\mathrm{ZnO}$ nanorods on Au electrodes for the development of an enzymatic glucose biosensor.

Ochratoxin-A (7-[L- $\beta$-phenylalanylcarbonyl]-carboxyl5-chloro-8-hydroxy-3,4-dihydro-3R-methylisocumarin, OTA) is one of the most abundant food-contaminating mycotoxins. ${ }^{53-57}$ OTA is found in tissues and organs of animals, including human blood and breast milk, and is known to produce nephrotoxic, tetratogenic, carcinogenic, and immune toxic activity in several animal species. OTA contamination has been reported in cereals, coffee, wines, dried fruits, and animal feeds, as well as in tissues and blood of animals and human beings. ${ }^{55,56,58}$ It affects humans mainly through consumption of improperly stored food products, and causes carcinogenicity. The International Agency of Research on Cancer has classified OTA as a possible carcinogenic compound (Group 2B, possibly by induction of oxidative DNA damage) for humans, since it causes immunosuppression and immunotoxicity. 55,56

Nano-ZnO film has been deposited onto ITO glass plate for co-immobilization of $r-\operatorname{IgGs}$ and bovine serum albumin (BSA) for OTA detection. The results of x-ray diffraction studies reveal the formation of Nano- $\mathrm{ZnO}$ with average particle size $\approx 5.0 \mathrm{~nm}$. Fourier transform infrared spectroscopy, SEM, and electrochemical impedance spectroscopy techniques have been used to characterize the Nano- $\mathrm{ZnO} /$ ITO electrode and the BSA/r-IgGs/Nano-ZnO/ITO immunoelectrode. The electrochemical impedimetric response of the BSA/r-IgGs/Nano-ZnO/ITO immunoelectrode, obtained as a function of OTA concentration, exhibits linearity as $0.006-0.01 \mathrm{nM} / \mathrm{dm}^{3}$, detection limit of $0.006 \mathrm{nM} / \mathrm{dm}^{3}$, response time of $25 \mathrm{~s}$, and sensitivity of $189 \Omega / \mathrm{nM} / \mathrm{dm}^{3} \mathrm{~cm}^{-2}$, with a regression coefficient of $0.997 . .^{55}$

\section{Urea nanosensors}

Urea is widely distributed in nature, and its analysis is of considerable interest in clinical and agricultural chemistry. ${ }^{60,61}$ It is known to be an important marker for evaluating uremic toxin levels. The normal level of urea in serum is from 15 to $40 \mathrm{mg} / \mathrm{dL}(2.5-7.5 \mathrm{mM} / \mathrm{l})$. In patients suffering from renal insufficiency, urea concentrations in serum vary from 180 to $480 \mathrm{mg} / \mathrm{dL}$ and, at elevated levels above $180 \mathrm{mg} / \mathrm{dL}$, hemodialysis is required. Apart from clinical applications, there is a growing demand for robust, reliable instrumentation toward the estimation of urea in other fields (eg, food science and environmental monitoring). As the principal component of nonprotein nitrogen in cow milk, urea is utilized as an indicator of protein-feeding efficiency. ${ }^{62}$ Nanopatterned porous alumina, prepared by electrical anodization in acid solution, has been used as an enzyme electrode and $\mathrm{pH}$ sensor for urea detection. The biocompatibility of nano-size porous alumina as an immobilization matrix for biocolloidal systems has stimulated interest for improved sensor sensitivity. The sensor was tailored to enlarge the active surface area, which in turn increased the sensitivity of the sensor. Some other supports used for urea biosensor fabrication include bilayer lipid membranes ${ }^{63}$ carbon paste electrodes, ${ }^{64}$ composite hydrogel membranes ${ }^{65}$ gelatin membranes, ${ }^{66}$ inorganic matrices (eg, clays, laponite, and 
zinc-aluminum-layered double hydroxide matrices), ${ }^{67}$ silicon dioxide $\left(\mathrm{SiO}_{2}\right)$ films, glass beads, diatomite, silica, porous glass, hornblende, and molecular sieves. ${ }^{68}$

Srivastava et $\mathrm{al}^{66}$ have described a urea biosensor that utilizes urease immobilized on gelatin beads. The system was successful in achieving a long storage stability, with a half-life of 240 days. The characteristics of this sensor include a detection limit from 0.8 to $23 \mathrm{mM}$ and a response time of $6 \mathrm{~min}$. Another sensing system has been proposed for urease immobilization on porous glass beads. ${ }^{69}$ The sensor setup proposed by Seki's group ${ }^{70}$ used urease immobilized on $\mathrm{SiO}_{2}$ films as the $\mathrm{pH}$-sensitive layer in light-addressable potentiometric sensors, with a detection range from 5-15 mM. However, more studies must be done in this field to fabricate a cost-effective, commercial sensor using these novel materials. The use of enzyme mixtures can provide improved performance in urea sensors. Seo et $\mathrm{al}^{71}$ determined ammonia via amperometric methods, using the immobilization of L-glutamate dehydrogenase on the immobilon-AV affinity membrane. Incorporation of the enzyme into a carbon paste was performed by Yang et $\mathrm{al}^{64}$ for the construction of an amperometric enzyme electrode. The enzyme-modified carbon paste electrode resulted in improved specificity and sensitivity. This system made use of bi-enzyme systems involving glutamate dehydrogenase and urease for urea estimation (Figure 3).

\section{Nanoparticles in bioassays Human immunoglobulin $\mathrm{G}$}

Bioassays are widely used in medical applications for sensitive and specific detection of biomolecules such as antibodies and antigens. The enzyme-linked immunosorbent assay (ELISA) is a well-known and commonly used tool for this purpose. ${ }^{72}$ However, in typical ELISA systems, several washing steps are performed that lead to an increased risk of, for example, residual contamination or loss in reagent, thereby reducing the apparent sensitivity, accuracy, and/or reliability.

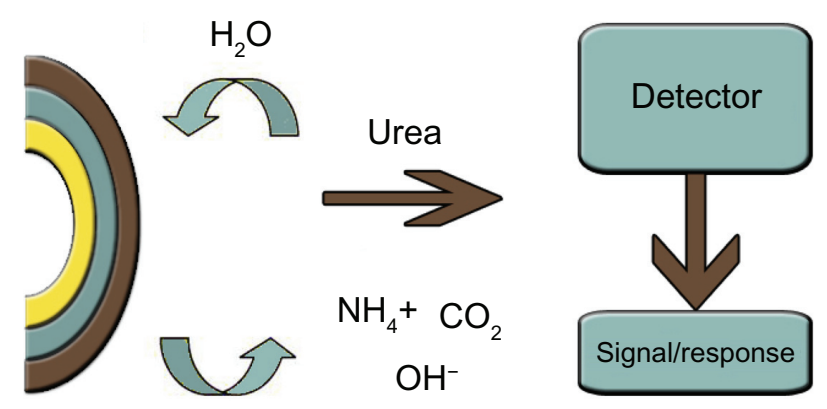

Figure 3 Schematic representation of a urea biosensor. ${ }^{66}$
Moreover, ELISA measurements give a static picture of the sample in terms of concentration of analyte, but provide no information about the binding kinetics or the dynamics of the reactions. A new approach for bioassays based on frequencyand time-domain measurements of magnetic nanoparticles (MNP) was recently introduced by Oisjoen et al. ${ }^{73}$ They demonstrate a one-step wash-free bioassay measurement system capable of tracking biochemical binding events. They combine the high resolution of frequency- and high speed of time-domain measurement in a single device in combination with a fast one-step bioassay. The one-step nature of their magnetic nanoparticle-based assay reduces the time between sample extraction and quantitative results, while mitigating the risks of contamination related to washing steps. The biofunctionalized MNPs they use for their experiment are multicore particles containing single domains of cobalt ferrous oxide. According to the manufacturer, the MNP system has a median hydrodynamic radius of $50 \mathrm{~nm}$. The size distribution is a critical factor in determining the sensitivity of this system. Larger MNPs have smaller relative change in hydrodynamic radii associated with molecules binding to their surfaces. The particles are functionalized with streptavidin, a biomolecule that has high affinity for biotin. According to specification from the provider, the MNPs can bind $80 \mathrm{pmol}$ of biotinylated protein per mg of MNPs, which corresponds roughly to 30 poly(sebacic anhydride) (PSA) molecules per MNP. This system has an advantage in that it does not require a separation step, which is usually employed for PSA detection through Brownian relaxation measurements of functionalized MNPs. ${ }^{73}$ There is no potential loss of signal associated with competitive binding of free analyte. This system has a sensitivity to $2 \mu \mathrm{L}$ samples, which means that less sample volume from a patient is needed, which provides the capability to perform additional parallel screening-type measurement on a single sample.

Many immunoassay techniques have been developed. Some substances such as radioisotopes, enzymes, chemiluminescent, and fluorescent compounds have been used as labels in immunoassay. However, water-soluble cadmium tellurium ( $\mathrm{CdTe}$ ) nanocrystals have attracted considerable attention as novel biological luminescent labels, compared to the traditional labels, in recent years. They have some unique physical and chemical properties, such as excellent brightness, narrow and precise, tunable emission, negligible photobleaching, fairly high quantum yields, and good chemical stability. Lately, some luminescent nanoparticles are also becoming very attractive for immunoassays. Crystalline europium oxide 
nanoparticles ${ }^{74}$ water-soluble poly (acrylic acid) grafted luminescent silicon nanoparticles ${ }^{75}$ and cadmium/selenium-zinc/sulphur core-shell quantum $\operatorname{dots}^{76}$ have been successfully applied as fluorescence labels in fluoroimmunoassays. The experimental procedure for determination of h-IgG using CdTe is very simple, involving the mixing of ten microliters of an analyte h-IgG with $0.8 \mathrm{ml}$ of iron oxide $\left(\mathrm{Fe}_{3} \mathrm{O}_{4}\right)$ and primary antibody solution. The mixture was incubated for $30 \mathrm{~min}$ at $37^{\circ} \mathrm{C}$. The $\mathrm{Fe}_{3} \mathrm{O}_{4} /$ primary antibody/h-IgG conjugate was formed, and then $2.0 \mathrm{~mL}$ of CdTe-secondary antibody conjugate was added into the tube to form the sandwich-type complex. Li et al ${ }^{77}$ were able to use this technique and show that the sandwich-type complex in an immunoassay can be sensitively detected by colloidal CdTe nanoparticles as labels. The CdTe label has better sensitivity and reproducibility than the traditional fluorescent labels. In addition, the CdTe nanoparticle labeling procedure is very simple, and the biochemical activity of the labeled compound is almost unaffected. Dextran- $\mathrm{Fe}_{3} \mathrm{O}_{4}$ magnetic nanoparticles were used in the immunoassays, so the separation procedure is simple and rapid. This new technique may be applied in many types of antibody-antigen system.

Surface-enhanced Raman scattering (SERS)-based immunoassay, a new technique with high sensitivity, showing the ability to detect picomole to femtomole amounts, is known as a potent detection means for protein determination. Raman reporter-labeled immuno-gold nanoparticles are utilized, and the biologic proteins are finally assayed qualitatively or quantitatively by the characteristics SERS peaks of the reporter. ${ }^{78,79}$ It is well known that the response ability of the SERS-based immunoassay is critically related to the size of the gold nanoparticles, as well as the aggregation degree of the immune aggregates. ${ }^{80,81}$ Beermann et a ${ }^{82}$ have investigated the surface enhancement ability of an isolated gold nanoparticle and found that a single nanoparticle with an average dimension of $25 \mathrm{~nm}$ was insufficient to enhance the Raman signals of the reporter. Similarly, it was discovered that no signal could be detected using small gold nanoparticles as a substrate. ${ }^{77}$ Conversely, bigger gold nanoparticles will result in greater SERS signals. But their stability may decrease significantly with the increasing sizes when they are modified with Raman reporters. ${ }^{82}$ Thus, taking into account the higher stability, many researchers have to carry out the SERS-based immunoassay with small gold nanoparticles. Meanwhile, an additional treatment (eg, silver stain) for SERS activation must be executed to ensure immunoassay with higher sensitivity. ${ }^{83-85}$
A highly sensitive immunoassay based on SERS has been developed with a novel immune marker, a Raman reporter-labeled immuno-gold aggregate on a SERS-active immune substrate. The features of those immune aggregates were characterized by UV-vis extinction spectra, transmission electron microscope images, SEM pictures, and SERS spectra. It is found that stable gold aggregates in appropriate morphologies can be induced by mixing proper amounts of reporter molecules with gold nanoparticles. Based on those reporter-labeled $\mathrm{Au}$ aggregates, immune aggregates with high stability can be prepared successfully by immobilizing antibody to the surface of the aggregates. Using this proposed immunoassay structure, the concentration detection of h-IgG was performed, and a calibration curve was obtained in the range from $100 \mathrm{ngmL}^{-1}$ to $100 \mathrm{fgmL}^{-1}$. This opens a new avenue for sensitive immunoassay and other biochemical analysis based on SERS.

\section{Steroids}

Steroids are lipid compounds. With the exception of cholesterol, steroids are natural hormones or hormone precursors. The determination of the levels of steroid hormones is an important issue for the inspection of endocrinological disorders related to adrenal or gonadal function. Among analytical methods used to determine the concentrations of steroid hormones or their precursors are immunoassays, ${ }^{86-88}$ fluorescence resonance energy transfer, ${ }^{89} \mathrm{SPR},{ }^{90}$ gas chromatography-mass spectrometry (GC/MS), ${ }^{91}$ and liquid chromatography-mass spectrometry (LC/MS) ${ }^{92-94}$ A sensitive assay for hormone detection that typically involves a mass spectrometer coupled with either a gas chromatograph or a liquid chromatograph has been developed by Fenlon et al. ${ }^{95}$ For these hormones, like anabolic steroids, according to the differences in fragmentation caused by collisions of medium energy related to the structure, it is difficult to find a common product ion or neutral loss. Furthermore, not only do most ELISA-like assays lack the sensitivity required to determine $>90 \%$ of hormone derivatives, ${ }^{94}$ but also these analytical procedures might introduce artefacts. The detection limits of the above methods range from $\mathrm{ng} / \mathrm{mL}$ to $\mathrm{pg} / \mathrm{mL} .{ }^{96}$ One-dimensional nanostructures such as carbon nanotubes and silicon nanowires (SiNW) have been demonstrated to be sensitive chemical and biological sensors. ${ }^{78}$ That detection results from the disturbance of charge on the surface of the functionalized nanostructure on which the target molecules are specifically recognized. For instance, the real-time detection of various antigens, ${ }^{97}$ oligonucleotides, ${ }^{98,99}$ proteins, ${ }^{100,101}$ and charged small molecules has been shown to be feasible on devices 
using nanowire or carbon-nanotube transistors as active transducers. The sensing mechanism in an electrically-based biosensor relies on an altered conductance or threshold voltage induced by the attachment of the charged analytes. Chang et $\mathrm{al}^{102}$ attempted to integrate protein engineering with the sensitive nature of a SiNW-field-effect transistor (FET) in charge disturbance to overcome the intrinsic weakness of SiNW-FET in detecting uncharged analytes. They chose an uncharged steroid, 19-norandrostenedione, as the target analyte. Delta 5-3-ketosteroid isomerase has served as a receptor for steroid recognition because of its well understood enzyme function. The primary concept of the sensing mechanism is based on intramolecular binding of a charged ligand, functioning as a reporter, to mimic the binding of an analyte to a protein. The major driving force favoring this association is generally thought to be the hydrophobic effect that prompts the hydrophobic ligand to bind with the protein. The thermodynamics of protein-ligand binding can be altered by a favorable control of enthalpy and, particularly in this model, the characteristic "entropy-driven" thermodynamic signature of the steroid. The analyte might replace the pre-situated ligand, which becomes thus exposed to the surface of the SiNW and perturbs the charge density and conductance of the nanostructure. In the presence of a steroid, the negatively charged 5-(2-aminoethylamino)1-naphthalenesulfonic acid (1,5-EDANS) moiety, which presumably occupies the steroid-binding site, is expelled and exposed to the nanowire surface (Figure 4). The electrical response produced from the 1,5-EDANS moiety is measured and the concentration is calculated accordingly. The sensitivity of this novel nano-bio-device can attain a femtomolar level. ${ }^{102}$

\section{C-reactive protein}

Recently a label-free quartz crystal microbalance (QCM) immunosensor for an important biomarker, C-reactive protein
(CRP) ${ }^{103-105}$ related with coronary heart disease, hypertension, and inflammation, has been developed with picomolar sensitivity. ${ }^{106}$ However, a possible matrix effect caused by serum viscosity might be present, like coloring substances in the case of ELISA. ${ }^{107}$ One way to avoid it is to dilute samples, including serum and plasma, to the degree at which sample viscosity approaches that of the reaction buffer. Recently, Kim et al ${ }^{107}$ conspicuously increased the sensor signal, and thus sensitivity of a label-free QCM immunosensor for CRP, by introducing a streptavidin-coated gold nanoparticle during antibody-antigen complexation, based on an indirect competitive assay format. When $200 \mu \mathrm{L}$ of the modified antibody, having the concentration of $0.250 \mathrm{mg} / \mathrm{mL}$, was added with GNp to the immunosensor system, the frequency shift obtained was $139.8 \pm 0.3 \mathrm{~Hz}$. Compared to the frequency shift of $91.1 \pm 1.3 \mathrm{~Hz}$ found with the addition of the unmodified antibody only, the signal augmentation after GNp binding amounted to $53.4 \%$, which resulted in sensitivity improvement of the immunosensor.

\section{Conclusion}

Clinicians, food technologists, and environmentalists all have an interest in generally increased sensitivity and limits of detection for a range of analytes. While the precise demands to meet today's requirements may be modest in these respects, few would contest the longer term benefits of reliable detection of trace amounts of various diagnostic indicators, additives, or contaminants.

As chemical analysis becomes simpler and more widely available, we can expect more accurate diagnosis and prognosis for different diseases. Nanosensors, capable of providing data through unique technology, could find wide application in monitoring our personal health, the food we eat, and our environmental health. The performance of nano-biochemical sensors is excellent in terms of sensitivity, selectivity, linearity, stability, response time, and reproducibility compared to

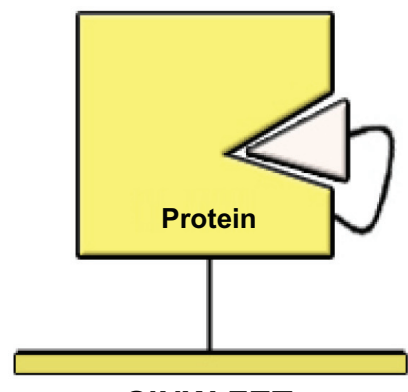

SiNW-FET

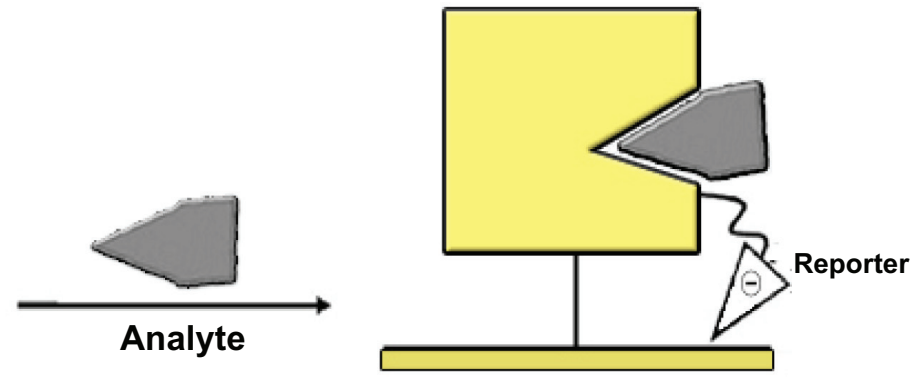

SiNW-FET

Figure 4 SiNW-FET platform for the detection of uncharged steroid-related analyte. ${ }^{105}$ Abbreviation: SiNW-FET, silicon nanowire field-effect transistor. 
the traditional biosensors. Nanoparticle labeling procedure is very simple, and the biochemical activity of the labeled compound is almost unaffected. This new approach is critically useful in preventing interference between chemically related analytes.

The use of core-shell NPs for the biocatalytic transformation of an enzyme could form the basis for the fabrication of an optical sensor for redox compounds. Moreover, the low $\mathrm{Km}^{\text {app }}$ value recorded when enzymes were immobilized on nano-biosensors indicates high affinity of sensors to analytes, which is interesting and promising for biosensor electrode construction.

\section{Disclosure}

The authors report no conflicts of interest in this work.

\section{References}

1. Murphy CJ, Jana NR. Controlling the aspect ratio of inorganic nanorods and nanowires. Adv Mater. 2002;14:80.

2. Sun Y, Xia Y. Shape-controlled synthesis of gold and silver nanoparticles. Science. 2002;298:2176.

3. Balasubramanian B, Kraemer KL, Reding NA, Skomski R, Ducharme S, Sellmyer DJ. Synthesis of monodisperse TiO(2)-paraffin core shell nanoparticles for improved dielectric properties. ACS Nano. 2010;4(4): 1893-1900.

4. van Dijk MA, Tchebotareva AL, Orrit M, et al. Absorption and scattering microscopy of single metal nanoparticles. Phys Chem Chem Phys. 2006;8:3486.

5. Ling J, Cheng ZH, Yuan FL, et al. Light scattering signals from nanoparticles in biochemical assay, pharmaceutical analysis and biological imaging. Trends Analyt Chem. 2009;28:4.

6. Thanh NT, Rosenzweig Z. Development of an aggregation-based immunoassay for anti-protein A using gold nanoparticles. Anal Chem. 2002;74(7):1624-1628.

7. Jiang ZL, Sun SJ, Liang AH, Liu CJ. A new immune resonance scattering spectral assay for trace fibrinogen with gold nanoparticle label. Anal Chim Acta. 2006;571(1):200.

8. Jiang Z, Huang W, Li J, Liang A, Zhang S, Chen B. Nanogold catalysisbased immunoresonance-scattering spectral assay for trace complement component 3. Clin Chem. 2008;54(1):116-123.

9. Clark LC, Lyons C. Electrode systems for continous monitoring in cardiovascular surgery. Ann N Y Acad Sci. 1962;102:1555-1582.

10. Ianniello RM, Yacynyeh AM. Carbon nanotube/chitosan/gold nanoparticles-based glucose. Anal Chem. 1981;53:2090.

11. Wang Y, Wanzhi W, Xiaoying L, Xiandong Z. Carbon nanotube/chitosan/ gold nanoparticles-based glucose biosensor prepared by a layer-by-layer technique. Mater Sci Eng C. 2009;29(1):50-54.

12. Yu J, Yu D, Zhao T, Zeng B. Development of amperometric glucose biosensor through immobilizing enzyme in a Pt nanoparticles/mesoporous carbon matrix. Talanta. 2008;74(5):1586-1591.

13. Meissam N, Motlagh K, Aboozar T. Preparation of silver hexacyanoferrate nanoparticles and its application for the simultaneous determination of ascorbic acid, dopamine and uric acid. Talanta. 2010;80(5):1657-1664.

14. Soundappa T, Hsuan T, Shen-Ming Chen T. Easy modification of glassy carbon electrode for simultaneous determination of ascorbic acid, dopamine and uric acid. Biosens Bioelectron. 2009;24(8):2712-2715.

15. Sofroniew MV, Priestley JV, Consolazione A, Eckenstein F, Cuello AC. Cholinergic projections from the midbrain and pons to the thalamus in the rat, identified by combined retrograde tracing and choline acetyltransferase immunohistochemistry. Brain Res. 1985;329(1-2):213-223.
16. Panfili G, Manzi P, Compagnone D, Scarciglia L, Palleschi G. Rapid assay of choline in foods using microwave hydrolysis and a choline biosensor. J Agric Food Chem. 2000;48(8):3403-3407.

17. Shimomura T, Itoh T, Mizukami F, Ono M. Amperometric determination of choline with enzyme immobilized in a hybrid mesoporous membrane. Talanta. 2009;78(1):217-220.

18. Dai H, ChiY, Wang Y, Wei M, Chen G. Biocompatible electrochemiluminescent biosensor for choline based on enzyme/titanate/chitosan composite modified electrode. Biosens Bioelectron. 2010;25(6):1414-1419.

19. Wang J, Musamaeh M. Carbon nanotube/teflon composite electrochemical sensors and biosensors. Anal Chem. 2003;75(9):2075-2079.

20. White A, Handler P, Smith EL, Hill RL, Lehman IR, editors. Principles of Biochemistry. 9th ed. New York, NY: McGraw-Hill; 1978.

21. Han J. Adsorption and electrochemical oxidation behaviour of NADH at a clean platinum electrode. J Electroanal Chem.1980;110:295-302.

22. Kotzian P, Janku T, Kalcher K, Vytras K. Catalytic activity of iron hexacyanoosmate (II) towards hydrogen peroxide and nicotinamide adenine dinucleotide and its use in amperometric biosensors. Anal Chim Acta. 2007;599(2):287-293.

23. Tang L, Zeng G, Shen G, et al. Highly sensitive sensor for detection of $\mathrm{NADH}$ based on catalytic growth of Au nanoparticles on glassy carbon electrode. Anal Bioanal Chem. 2009;393(6-7):1677-1684.

24. Gopalan A, Ragupathy D, Kim HT, Manesh KM, Lee KP. Pd (core)-Au (shell) nanoparticles catalyzed conversion of NADH to NAD ${ }^{+}$by UVvis spectroscopy - a kinetic analysis. Spectrochim Acta A Mol Biomol Spectrosc. 2009;74(3):678-684.

25. Valden M, Lai X, Goodman DW. Onset of catalytic activity of gold clusters on titania with the appearance of nonmetallic properties. Science. 1998;281(5383):1647-1650.

26. Artiss JD, Karcher RE, Cavanagh KT, et al. A liquid-stable reagent for lactic acid levels. Am J Clin Pathol. 2000;114:139-143.

27. Suzuk M, Akaguma H. Chemical cross-talk in flow-type integrated enzyme sensors. Sens Actuators B Chem. 2000;64:136-141.

28. Parra A, Casero E, Vazquez L, Pariente F, Lorenzo E. Design and characterization of a lactate biosensor based on immobilized lactate oxidase onto gold surfaces. Anal Chim Acta. 2006;555:308-315.

29. Marzouk SAM, Cosofret VV, Buck RP, Yang H, Cascio WE, Hassan SSM. A conducting salt-based amperometric biosensor for measurement of extracellular lactate accumulation in ischemic myocardium. Anal Chem. 1997;69:2646-2652.

30. Haccoun J, Piro B, Tran LD, Dang LA, Pham MC. Reagentless amperometric detection of L-lactate on an enzyme-modified conducting copolymer poly(5-hydroxy-1,4-naphthoquinone-co-5-hydroxy-3thioacetic acid-1,4-naphthoquinone). Biosens Bioelectron. 2004;19: $1325-1329$.

31. Palmisano F, Rizzi R, Centonze D, Zambonin PG. Simultaneous monitoring of glucose and lactate by an interference and cross-talk free dual electrode amperometric biosensor based on electropolymerized thin films. Biosens Bioelectron. 2000;15(9-10):531-539.

32. Suman S, Singhal R, Sharma AL, Malthotra BD, Pundir CS. Development of a lactate biosensor based on conducting copolymer bound lactate oxidase. Sens Actuators B Chem. 2005;107:768-772.

33. Trojanowicz M, Geschke O, Krawczyk TKV, Cammann K. Biosensors based on oxidases immobilized in various conducting polymers. Sens Actuators B Chem. 1995;28:191-199.

34. Park TM, Iwuoha EI, Smyth MR, Freaney R, McShane AJ. Sol-gel based amperometric biosensor incorporating an osmium redox polymer as mediator for detection of L-lactate. Talanta. 1997;44(6):973-978.

35. Lowinsohn D, Bertotti M. Flow injection analysis of blood L-lactate by using a Prussian Blue-based biosensor as amperometric detector. Anal Biochem. 2007;365:260-265.

36. Palmisano F, Quinto M, Rizzi R, Zambonin PG. Flow injection analysis of L-lactate in milk and yogurt by on-line microdialysis and amperometric detection at a disposable biosensor. Analyst. 2001;126(6):866-870.

37. Turner APF, Karube I, Wison GS, editors. Biosensors:fundamentals and applications. Oxford, UK: Oxford Science; 1987. 
38. Rahman MM, Shiddiky MJ, Rahman MA, Shim YB. A lactate biosensor based on lactate dehydrogenase/nictotinamide adenine dinucleotide (oxidized form) immobilized on a conducting polymer/ multiwall carbon nanotube composite film. Anal Biochem. 2009;384: 159-165.

39. Caplan LA, Pesce AJ. Clinical Chemistry: theory, analysis, correlation St Louis, MO: Mosby; 1989.

40. Avramoglu R, Basiciano K, Adeli HK. Lipid and lipoprotein dysregulation in insulin resistant states. Clin Chim Acta. 2006;368:1-19.

41. Vijayalakshmi A, Tarunashree Y, Baruwati B, et al. Enzyme field effect transistor (ENFET) for estimation of triglycerides using magnetic nanoparticles. Biosens Bioelectron. 2008;23:1708-1714.

42. Reddy RRK, Chadha A, Bhattacharya E. Porous silicon based potentiometric triglyceride biosensor. Biosens Bioelectron. 2001;16:313-317.

43. Bucolo G, David H. Quantitative determination of serum triacylglycerols by use of enzymes. Clin Chem. 1973;19:476-482.

44. Fossati P, Prencipe L. Serum triglycerides determined colorimetrically with an enzyme that produces hydrogen peroxide. Clin Chem. 1982;28:2077-2080

45. Mendez AJ, Cabeza C, Hsia SL. A flurometric method for the determination of triglycerides in the nanomolar quantities. Anal Biochem. 1986;156:386-389.

46. McGowan MW, Artiss JD, Strandbergh DR, Zak B. A peroxidasecoupled method for the colorimetric determination of serum triglyceride. Clin Chem. 1983;29:538-542.

47. Solanki PR, Dhand C, Kaushik A, Ansari AA, Sood KN, Malhotra BD. Nanostructured cerium oxide film for triglyceride sensor. Sens Actuators B Chem. 2009;141:551-556.

48. Wei A, Suna XW, Wang JX, et al. Enzymatic glucose biosensor based on $\mathrm{ZnO}$ nanorod array grown by hydrothermal decomposition. Appl Phys Lett. 2006;89:123902.

49. Wang JF, Sun XW, Wei A, et al. Zinc oxide nanocomb biosensor for glucose detection. Appl Phys Lett. 2006;88:233106.

50. Ansari AA, Singh R, Sumana G, Malhotra BD. Sol-gel derived nanostructured zinc oxide film for sexually transmitted disease sensor. Analyst. 2009;134:997-1002.

51. Liu YL, Yang YH, Yang FH, Liu MZ, Shen LG, Yu QR. Nanosized flower-like $\mathrm{ZnO}$ synthesized by a simple hydrothermal method and applied as matrix for horseradish peroxidase immobilization for electrobiosensing. J Inorg Biochem. 2005;99:2046-2053.

52. Yu J, Ju H. Preparation of porous titania sol-gel matrix for immobilization of horseradish peroxidase by a vapor deposition method. Anal Chem. 2002;74:3579-3583.

53. Radi AE, Berbel XM, Lates V, Marty JL. Label-free impedimetric immunosensor for sensitive detection of ochratoxin A. Biosens Bioelectron. 2009;24:1888-1892.

54. Pfohl LA, Petkova BT, Chernozemsky IN, Castegnaro M. Balkan endemic nephropathy and the associated urinary tract tumors: review on etiological causes and the potential role of mycotoxins. Food Addit Contam. 2002;19:282-302.

55. Kuiper-Goodman T. Mycotoxins and phytotoxins in perspective. In: Miraglia M, van Egmond H, Brera C, Gilbert J, editors. Mycotoxins and Phycotoxins - developments in chemistry, toxicology and food safety. Fort Collins, CO: Alaken; 1998:25.

56. Zimmerli B, Dick R. Ochratoxin A in table wine and grape-juice: occurrence and risk assessment. Food Addit Contam . 1996;13(6):655-668.

57. Ansari AA, Kaushik A, Solanki PR, Malhotra BD. Sol-gel derived nanoporous cerium oxide film for application to cholesterol biosensor. Electrochem Commun. 2008;10:1246-1268.

58. Kaushik A, Solanki PR, Ansari AA, Ahmad S, Malhotra BD. Chitosaniron oxide nanobiocomposite based immunosensor for ochratoxin-A. Electrochem Commun. 2008;10:1364-1368.

59. Ansari AA, Kaushik A, Solanki PR, Malhotra BD. Nanostructured zinc oxide platform for mycotoxin detection. Bioelectrochemistry. 2010;77:75-81.

60. Chua KS. Serum urea analysis using the Beckman BUN Analyzer. J Clin Pathol. 1976;29:517-519.
61. Taufik M, Yusuff M, Haruna OA, Muhamad NM. Effect of mixing urea with humic acid and acid sulphate soil on ammonia loss, exchangeable ammonium and available nitrate. Am J Environ Sci. 2009;5(5): 588-591.

62. Carlsson J, Pehrson B. Responses in urea and true protein of milk to different scheme protien feeding. Acta Vet Scand. 1994;35:67-77.

63. Nikolelis DP, Siontorou CO. Bilayer lipid membranes for flow injection monitoring of acetylcholine, urea, and penicillin. Anal Chem. 1995;67(5):369-944.

64. Yang M, Yang Y, Liu Y, Shen G, Yu R. Platinum nanoparticles-doped sol-gel/carbon nanotubes composite electrochemical sensors and biosensors. Biosens Bioelectron. 2006;21:1125-1131.

65. Chen J, Chiu S. Composite hydrogel membrane for urease immobilization to enhance urea hydrolysis rate. Enzyme Microb Technol. 2000;26:359-367.

66. Srivastava PK, Kayastha AM, Srinivasan K. Characterization of gelatinimmobilized pigeonpea urease and preparation of a new urea biosensor. Biotechnol Appl Biochem. 2001;34:55-62.

67. Barhoumi H, Maaref A, Rammah M, et al. Insulator semiconductor structures coated with biodegradable latexes as encapsulation matrix for urease. Biosens Bioelectron. 2005;20:2318-2323.

68. Thornton D, Byrne MJ, Flynn A, Johnson DB. Studies on the immobilization of trypsin on sand. Biochem Soc Trans. 1974;2:1360.

69. Yoneyama K, Fujino Y, Osaka T, Satoh I. Amperometric. sensing system proposed urease immobilization on porous glass beads. Actuators. 2001;76:152-157.

70. Seki A, Ikeda S, Kubo I, Karube I. Biosensors based on light-addressable potentiometric sensors for urea, penicillin.and glucose. Anal Chim Acta. 1998;373:9-13.

71. Seo ML, Kim JS, Lee SS, et al. Iron oxide-chitosan nano biocomposite for urea sensor. J Chem Soc. 1993;37:937.

72. Keck LD, Skeeles JK, McNew RW. Antibody detection in matched chicken sera and egg-yolk samples by commercial enzyme-linked immunosorbent assay kits for Newcastle disease virus, infectious bronchitis virus, infectious bursal disease virus, and avian reovirus. Avian Dis. 1993;37(3):825-828.

73. Oisjoen F, Schneiderman JF, Prieto Astalan A, Kalabukhov A, Johansson C, Winkler D. A new approach for bioassays based on frequency- and time-domain measurements of magnetic nanoparticles. Biosens Bioelectron. 2010;25(5):1008-1013.

74. Feng J, Shan G, Maquieira A, et al. Functionalized europium oxide nanoparticles used as a fluorescent label in an immunoassay for atrazine. Anal Chem. 2003;75:5282-5286.

75. Li ZF, Ruckenstein E. Water-soluble poly (acrylic acid) grafted luminescent fluorescent silicon nanoparticles and their use as biological staining labels. Nano Lett. 2004;4:1465-1467.

76. Goldman ER, Mauro J. Conjugation of luminescent quantum dots with antibodies using an engineered adaptor protein to provide new reagents for fluoroimmunoassays. Anal Chem. 2002;74:841-847.

77. Li X, Wang L, Zhou C, Guan T, Li J, Zhang Y. Preliminary studies of application of CdTe nanocrystals and dextran- $\mathrm{Fe}_{3} \mathrm{O}_{4}$ magnetic nanoparticles in sandwich immunoassay. Clinica Chimica Acta. 2007;378:168-174.

78. Cui Y, Wei Q, Park H, Lieber CM. Nanowire nanosensors for highly sensitive and selective detection of biological and chemical species. Science. 2001;293:1289-1292.

79. Chen MH, Chuang YJ, Tseng FG. Self-masked high-aspect-ratio polymer nanopillars. Nanotechnology. 2008;19(50):505301

80. Wang G, Park HY, Lipert RJ, Porter MD. Mixed monolayers on gold nanoparticle labels for multiplexed surface-enhanced Raman scattering based immunoassays. Anal Chem. 2009;81(23):9643-9650.

81. Knauer M, Ivleva NP, Liu X, Niessner R, Haisch C. Surface-enhanced Raman scattering-based label-free microarray readout for the detection of microorganisms. Anal Chem. 2010;82(7):2766-2772.

82. Beermann J, Novikov SM, Leosson K, Bozhevolnyi SI. Surface enhanced Raman imaging: periodic arrays and individual metal nanoparticles. Opt Express. 2009;17(15):12698. 
83. Han XX, Kitahama Y, Itoh T, Wang CX, Zhao B, Ozaki Y. Proteinmediated sandwich strategy for surface-enhanced Raman scattering: application to versatile protein detection. Anal Chem. 2009;81(9): 3350-3355.

84. Song C, Wang Z, Zhang R, Yang J, Tan X, Cui Y. Highly sensitive immunoassay based on Raman reporter-labeled immuno-Au aggregates and SERS-active immune substrate. Biosens Bioelectron. 2009;25(4):826-831.

85. Chon H, Lee S, Son SW, Oh CH, Choo J. Highly sensitive immunoassay of lung cancer marker carcinoembryonic antigen using surfaceenhanced Raman scattering of hollow gold nanospheres. Anal Chem. 2009;81(8):3029-3034.

86. England BG, Parsons GH, Possley RM, McConnell DS, Midgley AR. Ultrasensitive semiautomated chemiluminescent immunoassay for estradiol. Clin Chem. 2002;48(9):1584-1586.

87. Taieb J, Benattar C, Birr AS, Lindenbaum A. Limitations of steroid determination by direct immunoassay. Clin Chem. 2002;48(3): 583-585.

88. Hungerford NL, Sortais B, Smart CG, et al. Analysis of anabolic steroids in the horse: development of a generic ELISA for the screening of 17alpha-alkyl anabolic steroid metabolites. J Steroid Biochem Mol Biol. 2005;96:317-334.

89. Macara IG, Lannigan DA. Novel biosensors for the detection of estrogen receptor ligands. J Steroid Biochem Mol Biol. 2005;96:235-244.

90. Toshie KT, Tomofum F, Tadashi O, et al. HX531, a retinoid X receptor antagonist, inhibited the 9 -cis retinoic acid-induced binding with steroid receptor coactivator-1 as detected by surface plasmom resonance. J Steroid Biochem Mol Biol. 2005;94:303-309.

91. Ventura R, Roig M, Perez B, et al. Detection of the administration of 17beta-nortestosterone in boars by gas chromatography/mass spectrometry. Rapid Commun Mass Spectrom. 2008;22:1863-1870.

92. Qin F, Zhao YY, Sawyer MB, Li XF. Hydrophilic interaction liquid chromatography-tandem mass spectrometry determination of estrogen conjugates in human urine. Anal Chem. 2008;80:3404-3411.

93. Pozo OJ, Eenoo PV, Thuyne WV, Deventer K, Delbeke FT. Direct quantification of steroid glucuronides in human urine by liquid chromatography-electrospray tandem mass spectrometry. J Chromatogr A. 2008;1183:108-118

94. Geisler J, Ekse D, Helle H, Duong NK, Lonning PE. An optimized, highly sensitive radioimmunoassay for the simultaneous measurement if estrone estradiol and estrone sulfate in the ultra-low range in human plasma samples. J Steroid Biochem Mol Biol. 2008;109:90-95.
95. Fenlon KA, Johnson AC, Tyler CR, Hill EM. Gas-liquid chromatography-tandem mass spectrometry methodology for the quantitation of estrogenic contaminants in bile of fish exposed to wastewater treatment works effluents and from wild populations. J Chromatogr A. 2010; 1217(1):112-118.

96. Al-Dujaili EA. Development and validation of a simple and direct ELISAmethod for the determination of conjugated (glucuronide) and non-conjugated testosterone excretion in urine. Clin Chim Acta. 2006;364:172-179.

97. Pal S, Alocilja EC, Downes PF. Nanowire labeled direct-charge transfer biosensor for detecting Bacillus species. Biosens Bioelectron. 2007;22:2329-2336

98. So HM, Won K, Kim YH, et al. Single walled carbon nanotube biosensors using aptamers as molecular recognition elements. J Am Chem Soc. 2005;127:11906-11907.

99. Kim DS, Jeong YT, Park HJ, et al. An FET-type charge sensor for highly sensitive detection of DNA sequence. Biosens Bioelectron. 2004;20:69-74.

100. Besteman G, Lee JO, Wiertz FGM, Heering HA, Dekker C. Enzymecoated carbon nanotubes as single-molecule biosensors. Nano Lett. 2003;3:727-730.

101. Patolsky F, Lieber CM. Nanowire nanosensors. Mater Today. 2005; 8:20-28.

102. Chang KS, Chen CC, Sheu JT, Li Y-K. Detection of an uncharged steroid with a silicon nanowire field-effect transistor. Sens Actuators B Chem. 2009;138:148-153.

103. Gabay C, Kushner IN. Acute-phase proteins and other systemic responses to inflammation. $N$ Engl J Med. 1999;340:448-454.

104. Rifai N, Ridker PM. High-sensitivity C-reactive protein: a novel and promising marker of coronary heart disease. Clin Chem. 2001;47:403-411.

105. Wolf M, Juncker D, Michel B, Hunziker P, Emmanuel D. Simultaneous detection of $\mathrm{C}$-reactive protein and other cardiac markers in human plasma using micromosaic immunoassays and self-regulating microfluidic networks. Biosens Bioelectron. 2004;19:1193-1202.

106. Kim N, Park IS, Kim WY. Salmonella detection with a direct-binding optical grating coupler immunosensor. Sens Actuators B Chem. 2007;121:606-615.

107. Kim N, Kim YJ, Cho DK. Gold nanoparticle-based signal augmentation of quartz crystal microbalance immunosensor measuring C-reactive protein. Curr Appl Phys. 2010;10(4):1227-1230.
Nanotechnology, Science and Applications

\section{Publish your work in this journal}

Nanotechnology, Science and Applications is an international, peerreviewed, open access journal that focuses on the science of nanotechnology in a wide range of industrial and academic applications. It is characterized by the rapid reporting across all sectors, including engineering, optics, bio-medicine, cosmetics, textiles, resource sustainability

\section{Dovepress}

and science. Applied research into nano-materials, particles, nanostructures and fabrication, diagnostics and analytics, drug delivery and toxicology constitute the primary direction of the journal. The manuscript management system is completely online and includes a very quick and fair peer-review system, which is all easy to use. 